\title{
LASER ATOMIC EMISSION SPECTROMETER WITH ACHROMATIC OPTICAL SYSTEM
}

\author{
E. S. Voropay, ${ }^{\text {a I. M. Gulis, }}{ }^{\text {a D. S. Tarasau, }},{ }^{\text {a,b, }}$, \\ K. F. Ermalitskaia, ${ }^{a}$ M. P. Samtsov, ${ }^{b}$ A. E. Radko, \\ A. P. Zajogin, ${ }^{a}$ K. A. Shevchenko, ${ }^{b}$ and A. A. Kirsanov ${ }^{a}$ \\ UDC 543.423
}

A laser atomic emission multichannel spectrometer (LAEMS) with an achromatic optical configuration is developed for research and educational purposes. The new spectrometer fits all the requirements for equipment for laser atomic emission spectroscopy, and has a number of advantages owing to the features of the design and technical characteristics of its components. The excitation source for the spectrometer includes two Nd:YAG lasers pumped by semiconductor laser diode arrays with a controllable energy (from 0 to $100 \mathrm{~mJ}$ ) and interval between between pulses (from 1 to $100 \mu \mathrm{s}$ ), and an average pulse duration of $\approx 15 \mathrm{~ns}$. The LAEMS provides for studies in both a single pulse and a double pulse laser ablation regime and excitation of emission spectra. Double pulse laser ablation on the LAEMS provides a substantial (up to a factor of 10) increase in the analytic signal with a negligible (1.5-2 times) increase in surface damage.

Keywords: laser ablation, laser atomic emission spectroscopy (laser induced breakdown spectroscopy), double laser pulses, spectrometer.

Introduction. Over the last two decades, laser atomic emission spectroscopy (laser induced breakdown spectroscopy) has been one of the most rapidly developing and widely used methods for qualitative and quantitative elemental analysis of objects [1-3]. The advantages of this method include its applicability to a wide class of objects, the possibility of analyzing, including remotely, solids as well as liquids, the small amounts of material needed for analysis $\left(10^{-10}-10^{-11} \mathrm{~g}\right)$, negligible surface damage (crater diameter $\leq 100 \mu \mathrm{m}$, with an evaporated layer of thickness from tenths of a $\mu \mathrm{m}$ ), and the lack of a need for preliminary mechanical and chemical preparation of the surface [1,3-5]. Laser atomic-emission spectroscopy is used to study biological objects (plants, lichens, samples, biological liquids, and human and animal tissues), industrial work pieces (analysis of raw materials as well as completed production), art objects, jewelry, artifacts, structural materials [1-10]. The optimum parameters of laser radiation for analysis of different kinds of objects have been determined on the basis of a large amount of experimental and theoretical work. Thus, the most widespread conditions for analytic work with laseratomic-emission spectroscopy involve $\mathrm{Nd}$ :YAG laser light with $\lambda=1064 \mathrm{~nm}$ (less often, $532 \mathrm{~nm}$ ), laser pulse durations of $10-15 \mathrm{~ns}$, and energies of 20-100 $\mathrm{mJ}[1,2,5,7]$.

A number of scientific studies have been devoted to the use of doubled laser pulses (DLP), separated by microsecond intervals between pulses, as the source for laser ablation of matter and spectral excitation. It has been shown that the transition from single- to double-pulse laser ablation for a fixed total energy and power of the radiation yields a manifold increase in the analytic signal, which can reduce the error in quantitative analysis [4, 7, 11]. Experimental studies of different DLP configurations, a scheme with a pre-ablation spark, and orthogonal and collinear configurations, have been discussed in $[1,2,5]$. The latter is optimal, since it provides the required result in the simplest technical form.

Despite a large number of scientific papers on laser atomic-emission spectroscopy, a substantial fraction of these studies are not done with prepared spectrometers, but on the basis of systems assembled from individual parts based on engineering laboratories and practical centers. These systems meet the requirements for the spectrometers but are significantly limited for use in teaching and for a making a large volume of measurements in various areas.

\footnotetext{
* To whom correspondence should be addressed.
}

${ }^{\mathrm{a} B e l a r u s i a n}$ State University, Minsk, 220030, Belarus; email: voropay@bsu.by; ${ }^{\mathrm{b}}$ A. N. Sevchenko Institute for Applied Physical Problems, Belarusian State University, Minsk, Belarus. Translated from Zhurnal Prikladnoi Spektroskopii, Vol. 88, No. 3, pp. 485-492, May-June, 2021. Original article submitted March 24, 2021. 
At the department of laser physics and spectroscopy of the physics faculty of the Belarusian State University (BGU) and in the spectroscopy laboratory of the A. N. Sevchenko Institute for Applied Physical Problems at BGU, a laser atomicemission multichannel spectrometer (LAEMS) has been developed for scientific and teaching purposes. The LAEMS has been developed using fundamental and design solutions aimed at bringing it closer to current requirements for equipment of this type in daily practice.

Operating Principle and Features of the Optical System. The general principle of obtaining spectra using laser atomic-emission spectroscopy involves recording the plasma emission from a plasma produced during interaction of a laser pulse with the surface of a sample using a polychromator spectrometer. In a two-pulse LAEMS each pulse from the DLP pair has a separate purpose. The first interacts with the target surface; this yields a plasma containing both excited atoms and ions, but also particles of the test material in liquid and solid phases. The second laser pulse interacts with the plasma, further exciting it and evaporating liquid droplets and solid particles. The vapor-gas cloud formed by the first laser pulse partially absorbs the radiation from the second pulse so only part of its energy reaches the target surface.

The design and functional features of two-pulse LAEMS are determined by the configuration for spatial combination of the DLP: orthogonal, with a pre-ablation spark, and collinear. An analysis showed that in LAEMS for teaching and scientific purposes it is appropriate to use a collinear combination of DLP, which is distinguished by relative simplicity of technical implementation and the ability to analyze a large class of objects. The high analytic signal level in this combination arises both from a greater amount of laser ablation and from additional excitation of the formed plasma by the second pulse. For a collinear configuration the two pulses fall in succession on the sample. Here the intensity of the spectral lines in analyses of solutions is increased because the first pulse forms a "cavitation bubble" on which the second pulse is focused before formation of the ablation plasma.

The optical configuration of the spectrometer is shown in Fig. 1. It was developed taking aberration-optical and technical-technological specifications into account. The spectrometer is equipped with two neodymium lasers pumped with semiconductor diode laser arrays 1 (developed at the Institute of Physics of the National Academy of Sciences of Belarus). Pumping with semiconductor diode laser arrays rather than flash lamps makes it possible greatly to increase the lifetime of the system and the stability of its output characteristics. The two-pulse laser generates DLP of duration $\leq 15 \mathrm{~ns}$ with $\lambda=1064 \mathrm{~nm}$ and a variable interval between them of $1-100 \mu$ s with energies of up to $100 \mathrm{~mJ}$ and a repetition rate for the pulse pairs of $<10 \mathrm{~Hz}$.

To ensure achromaticity of the focusing system for the laser light on the object 17 and the light collection and input system into the fiber-optic input 14 of the polychromator-spectrometers 18,19 for the plasma light it is proposed that mirror and off-axis paraboloids 11 and 16 be used instead of refractive objectives. The major disadvantage of the refractive objective traditionally used in apparatus for LAEMS is the difficulty of reaching an acceptable level of achromatism over a wide spectral range, including the UV (there is a limited choice of transparent materials). At the same time, the presence chromatic aberration leads to differences in the efficiency of delivering radiation to a fiber-optic cable for different spectral components of the plasma emission, which may show up as a source of errors in measurements of relative line intensities. The mirror objective 16 aimed at the objective 17 serves the function of focusing the exciting beam and the beam of the semiconductor targeting laser $7(\lambda=650 \mathrm{~nm})$ propagating coaxially with it, and also ensures collection of plasma light and forms an expanded parallel beam which is then focused onto the input of the fiber-optic by the mirror objective 11 . The laser beam is delivered to the objective 16 parallel to the principal optic axis of the paraboloid using the auxiliary plane mirror 15 with a normal oriented at an angle of $45^{\circ}$ relative to the indicated axis. The center of this mirror lies near the central axis of the mirror 16, which ensures invariability of the position of the center of the focal spot of the laser beam on the sample (target) surface as the sample is moved along the axis of the laser beam (e.g., in order to change the energy density on the target). The mirror 15 has a luminous diameter of $10 \mathrm{~mm}$ that is small compared to the diameter of the parabolic objective $(50 \mathrm{~mm})$, so that vignetting of the plasma emission collected by the parabolic objective does not exceed $10 \%$ when the shadowing caused by components of the holder of the small mirror is taken into account. As a compromise between the component specifications and the need to focus the laser light into a spot with a diameter of $50-100 \mu \mathrm{m}$, the focal distances of the objectives were chosen to be close to $100 \mathrm{~mm}$. Because of the large off-axis angle of the mirror 16, a distance of $\sim 70 \mathrm{~mm}$ from the protective quartz glass of the sample chamber to the surface of a sample is ensured.

The use of off-axis parabolic mirrors operating at off-axis angles of $90^{\circ}$ (mirror 16, effective focal length $101.6 \mathrm{~mm}$ ) and $30^{\circ}$ (mirror 11, effective focal length $136.1 \mathrm{~mm}$ ) imposes specifications on the parallelism of the focused laser beam to the principal axes of the parabolic mirrors. Calculations and preliminary experiments show that a deviation of $1^{\circ}$ or more from this parallelism leads to an unacceptable astigmatism, both at the focal spot on the sample surface and in the image of 


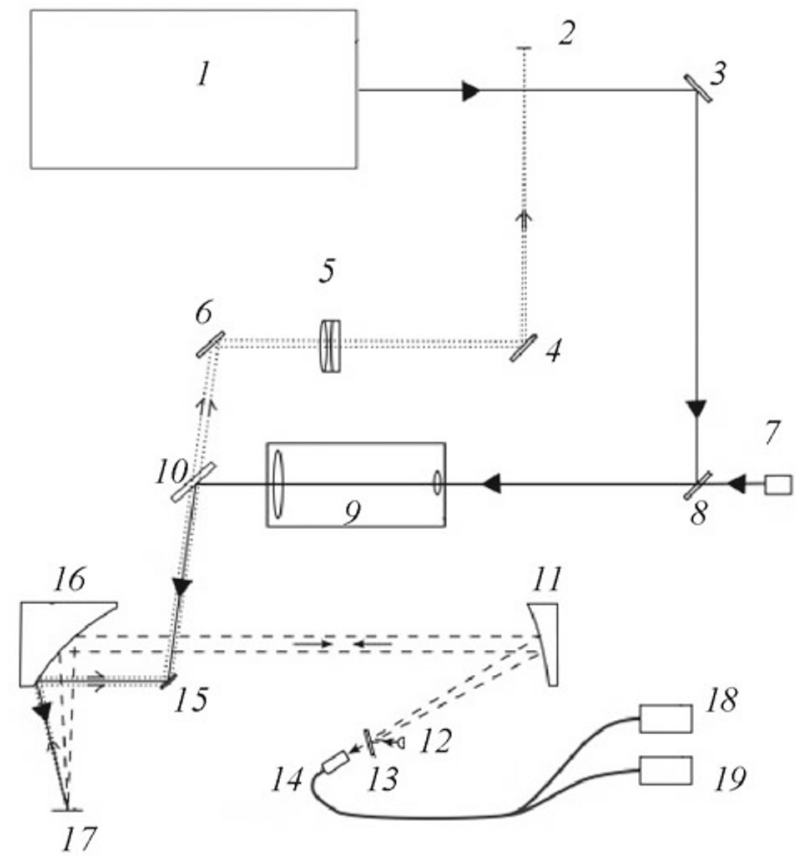

Fig. 1. Optical configuration of the LAEMS: 1, two-pulse laser; 2, video camera; 3, 4, 6, 15 , nonselective plane mirrors; 5 , imaging objective (observation channel); 7 , targeting laser; 8 , selective plane mirror; 9, telescope; 10 semitransparent plane mirror; 11, parabolic mirror (off-axis angle $30^{\circ}$ ); 12, illuminating LED; 13 , plane mirror (normally removed, installed for illumination of object when necessary; 14, fiber-optic input (to spectrometer); 16 , parabolic mirror (off-axis angle $90^{\circ}$ ); 17 , object; 18,19 , polychromators-spectrometers.

this spot on the input to the fiber-optic cable 14. The requirement of parallelism is easily met with simple autocollimation adjustment procedures. At the same time, a deliberate deviation of the laser beam from parallelism to the principal axis of the paraboloid (by inclination of the mirror 15) makes it possible to obtain focal spots in the form of astigmatic foci (of roughly elliptical shape). The length of the axes of the ellipse may range from a few to hundreds of microns, which makes it possible to vary the shapes and dimensions of the focal spots. This may be of interest in work with samples that have a complicated nonuniform surface structure and extend the choice of interaction regions.

To ensure the required spot diameter $(\leq 70 \mu \mathrm{m})$ focused on the sample for a two-pulse laser source with a divergence of $\sim 2 \mathrm{mrad}$, the laser beam is passed through a 5-times telescopic expander 9 that lowers the divergence to a level of $\sim 0.5 \mathrm{mrad}$. An auxiliary turning mirror 13 is placed in front of the entrance to the fiber-optic; when the mirror is introduced into the light beam the entrance to the fiber-optic is covered and the light from a green light diode 12 is fed into the optical system formed by the two parabolic mirrors to illuminate the target surface. The system that forms the image of the surface includes the parabolic mirror 16 with an off-axis angle of $90^{\circ}$, a long-focus (300 $\mathrm{mm}$ ) low-throughput objective 5, opaque $5,6,15$ and semitransparent 10 plane mirrors, as well as a video camera 2 .

The sample is placed on a table that moves along the beam with a linear low-profile positioner (manual movement) and in two directions perpendicular to the beam using linear motorized positioners, which enable computer controlled scanning of the sample surface. Spectrometers with CCD array detectors are used to record the plasma spectra: 18, based on an SDH-1 polychromator (ZAO "SOLAR Laser systems, range 190-800 nm and resolution $0.1 \mathrm{~nm}$ for a simultaneously detectable spectral interval of $150 \mathrm{~nm}$ ) and 19, a spectrometer with an original design described in [12].

The laser source operating regimes, sample scanning systems, and systems for recording the spectra are controlled by an original program which provides simultaneous control of all components of the LAEMS and the basic functions for preliminary processing, analyzing, and imaging the spectral data. In developing the original program in the $\mathrm{C}++$ language in a Microsoft Visual Studio 2005 medium, an SDK controller of the detection system of the ORMINS firm based on $\mathrm{CCD}$ sensors was used. The diffraction grating of the polychromator was controlled manually; the readings of the micrometer 
$I, 10^{4}$ rel. units

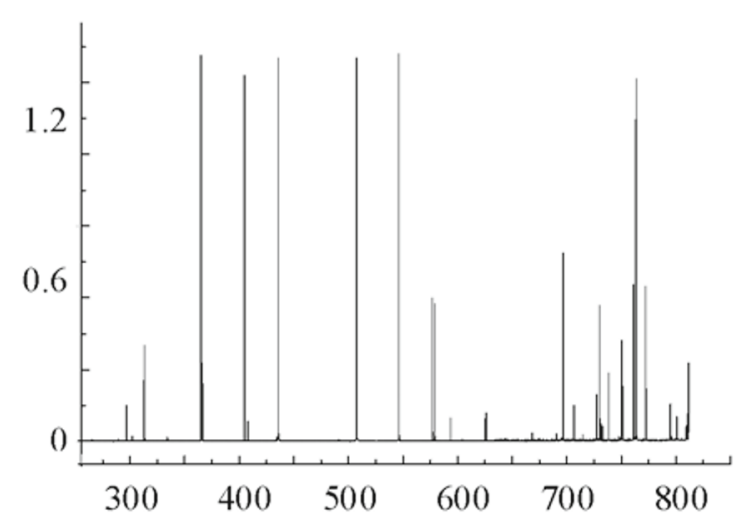

$a$

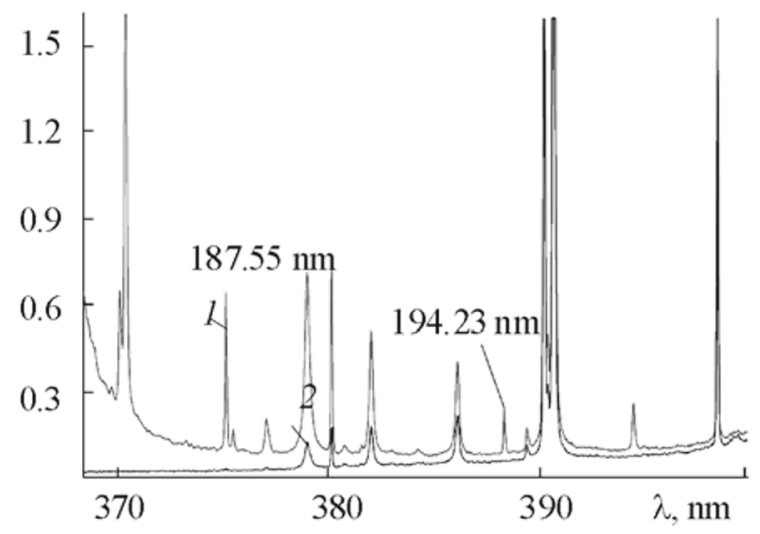

Fig. 2. Spectrum of a CAL-2000 mercury-argon lamp obtained by merger of spectra recorded with an SDH-I LAEMS spectrometer in several ranges (a) and the spectrum of a PRK-2 mercury lamp without (1) and with (2) a BS-4 optical filter taken with an SDH-I LAEMS spectrometer (b).

screw are used in the LAEMS program for calibration of the spectral range of the polychromator with the detection system.

The program offers the possibility of detecting spectra in four regimes: a single detection cycle for the spectrum of the background signal, a single cycle of detection the spectrum from one point, a single cycle of detection of the spectrum from points distributed over a specified surface, and a continuous cycle of detection of spectra from a single point without data retention. The operator of the LAEMS program can specify the pulse energies and time delay between the DLP, and examine and make a preliminary analysis of the resultant data. The advantage of the LAEMS program is the inbuilt data base of spectrum lines which can be used to create methods for qualitative and quantitative elemental analysis of samples (up to 50 determinable chemical elements).

Basic Optical-Technical and Analytic Characteristics of the LAEMS. The LAEMS was developed for science teaching at the physics faculty of the Belarusian State University. The design of the optical system and selection of the LAEMS spectrometer components were intended to ensure a technical level comparable to its modern domestic and foreign analogs. The major analytical parameters of the spectrometer are listed in Table 1.

The major spectral characteristics of the LAEMS are confirmed by recording the emission of light sources with known parameters. A CAL-2000 mercury-argon lamp (Fig. 2a) was used in the 253-811 nm range, a PRK-2 mercury lamp in the 190-260 nm range with detection in the second order of diffraction (Fig. 2b). The measurements show that the spectrometer operates in the necessary range with a spectral resolution of $0.09 \mathrm{~nm}$ (average value).

Application of the LAEMS in Scientific Research Activity. An LAEMS and a Linnik MII-4 microinterferometer have been used to study the interaction of single and double (DLP) laser pulses with a sample surface and the formation and propagation of an ablation plasma. It was found that for metals, going from single to double laser pulses for a fixed total energy and power leads to a substantial increase in the analytic signal by a factor of from 2 to 10 , depending on the energy of the laser pulses and the thermal properties of the material. Measuring the surface damage with the MII- 4 microinterferometer showed that here the diameter of the crater on the surface of metals and alloys is essentially unchanged and the thickness of the evaporated surface increases by a factor of 1.5-2.5. The calibration curves for quantitative analysis of multicomponent alloys remain linear (correlation coefficient $R^{2} \sim 0.90-0.97$ ). This circumstance makes the double pulse LAEMS regime preferable for studies of finished products, as well as of samples with an historical significance. The transition from single to double laser pulses makes it possible to reduce the number of pulses onto a point (by a factor of 2-10) without increasing the error in the analysis while retaining the detection sensitivity.

Methods for qualitative and quantitative microanalysis of artifacts and ancient coins have been developed based on the LAEMS, with recording of the emission spectra of real and false ancient coins, barbarian imitations of imperial Roman denarii found in the territory of Belarus and kept in collections at the numismatic cabinet of the Belarusian State University and in private collections; and gold-plated Roman coins, as well as fragments of jubilee decorations from the 
TABLE 1. Major Technical and Analytic Characteristics of the LAEMS Spectrometer

\begin{tabular}{|c|c|}
\hline Excitation source & $\begin{array}{l}\text { Two-pulse solid-state Nd:YAG laser } \\
\text { pumped with laser diode arrays }\end{array}$ \\
\hline Output wavelength, nm & 1064 \\
\hline Operating regimes & $\begin{array}{l}\text { single pulses } \\
\text { double pulses }\end{array}$ \\
\hline Pulse repetition rate, $\mathrm{Hz}$ & up to 10 \\
\hline Pulse duration, ns & $\sim 15$ \\
\hline Range of variation of pulse energy, $\mathrm{mJ}$ & $0-100$ \\
\hline Range of variation of delay between doubled laser pulses, $\mu \mathrm{s}$ & $1-100$ \\
\hline \multicolumn{2}{|l|}{ Polychromator with a detection system } \\
\hline Spectral range, $\mathrm{nm}$ & $190-800$ \\
\hline Spectral resolution (average), $\mathrm{nm}$ & 0.09 \\
\hline \multicolumn{2}{|l|}{ Sample chamber and visual observation system } \\
\hline Dimensions of analyzed samples, $\mathrm{mm}$ & to $200 \times 200 \times 200$ \\
\hline Diameter of laser beam on sample surface, $\mu \mathrm{m}$ & $\sim 50$ \\
\hline $\begin{array}{l}\text { Mechanism for coordinate positioning of sample holder: } \\
\qquad \begin{array}{l}X \text { axis } \\
Y \text { axis } \\
Z \text { axis }\end{array}\end{array}$ & $\begin{array}{l}\text { by program } \\
\text { manual } \\
\text { by program }\end{array}$ \\
\hline Accuracy of positioning on sample surface, $\mu \mathrm{m}$ & 100 \\
\hline Visual channel & Web-camera with objective \\
\hline $\begin{array}{l}\text { LAEMS program developed for control of laser sources, sample scanning and } \\
\text { spectral recording systems, for analysis and processing of spectral data }\end{array}$ & $\begin{array}{l}\text { Windows XP/Vista/7/10 Operating } \\
\text { system }\end{array}$ \\
\hline \multicolumn{2}{|c|}{$\begin{array}{l}\text { Operating regimes: } \\
\text { cle recording of background spectrum signal; } \\
\text { cycle recording of spectrum from one point; } \\
\text { g of spectra from points distributed over a specified area; } \\
\text { cording spectra from a single point without retention of data }\end{array}$} \\
\hline Number of determinable chemical elements & 50 \\
\hline
\end{tabular}

first millennium BC found in the territory of modern Belarus. Figure 3 shows a photograph of a Polotsk shilling with "traces of damage" from DLP, a fragment of a spectrum of this coin taken with an LAEMS, a photograph of a silver coin from the 16th century with "traces of damage," a photograph of a crater on the surface of the silver coin magnified by 300 times, and a photograph of a bronze ornament from the first millennium BC under analysis on the LAEMS with a showing "traces of damage."

The LAEMS that has been developed meets the requirements for spectrometers for surface, layered, and lowdestruction analysis, and permits taking full advantage of DLP for reducing the damage to sample surfaces without increasing the error in the analysis.

At the department of laser physics and spectroscopy in the physics faculty of the Belarusian State University a set of laboratory studies of laser atomic-emission spectroscopy has been undertaken for undergraduate and graduate students, including some studying in English. This set includes six laboratory projects employing an LAEMS. Each laboratory project is planned for six academic hours and has a distinct scientific-research approach. 

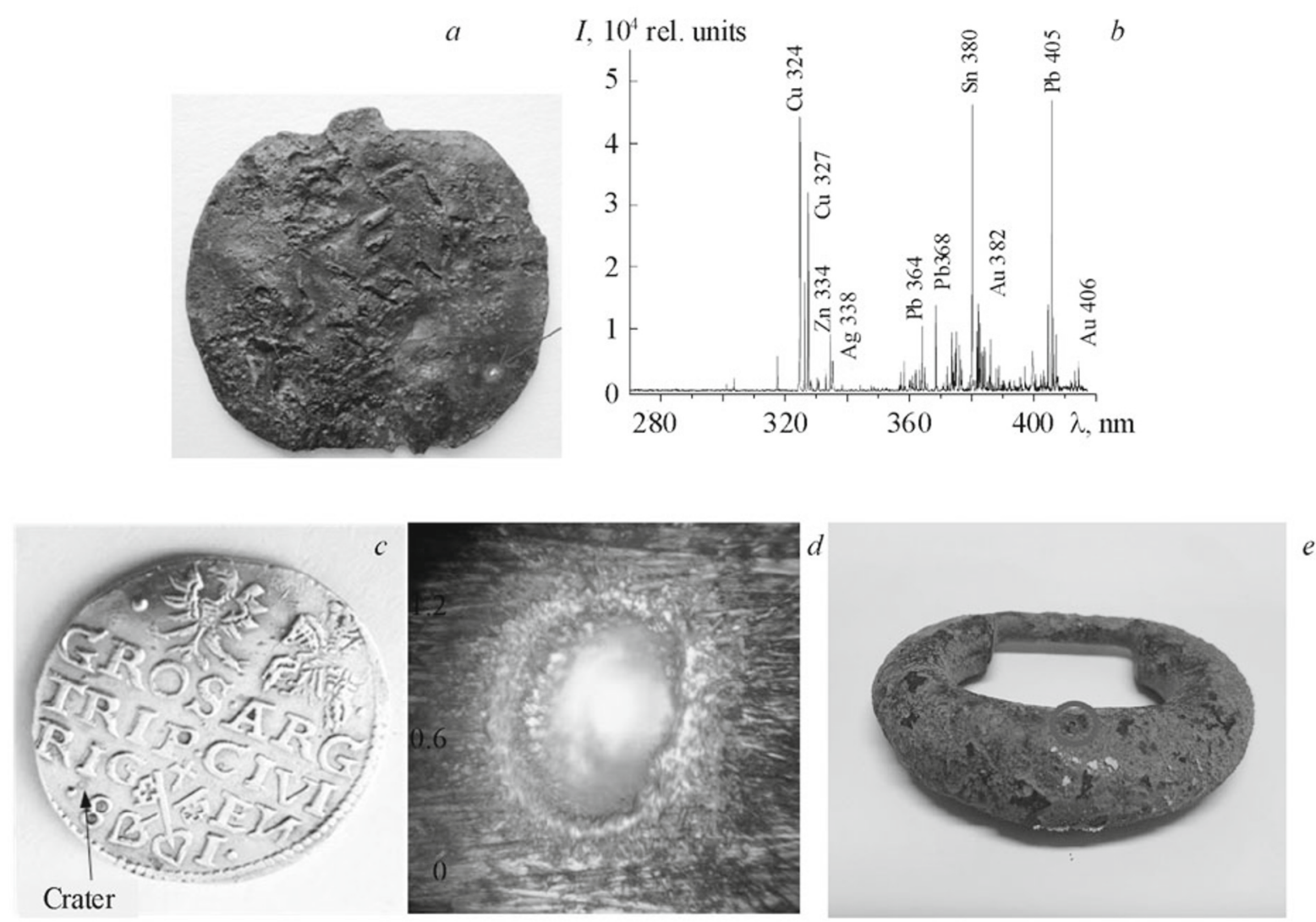

Fig. 3. A Polotsk shilling with "traces of damage" from double laser pulses (a), a fragment of a spectrum of this coin taken with an LAEMS (b), a silver coin from the 16 th century with "traces of damage" (c), a photograph of a crater on the surface of the silver coin magnified by 300 times (d), and a bronze ornament from the first millennium BC showing "traces of destruction" (e).

Conclusions. A scheme has been developed for a laser atomic-emission multichannel spectrometer (LAEMS) for research in atomic spectroscopy and a sample has been constructed. The use of a two-pulse laser with semiconductor diode rather than flashlamp pumping substantially increases the effective output of the system and the stability of its output characteristics. Because of this, the new spectrometer is accessible for teaching laboratories. The energies of the double pulses of this two-pulse laser can be set independently. This is not possible in lasers with flashlamp pumping. Another fundamental design solution in the laser atomic-emission multichannel spectrometer is the use of mirror objectives (off-axis paraboloids) instead of refractive objectives. This ensures achromaticity of the system for focusing the laser light on the object and of the system for light collection and delivery of the plasma light to the fiber-optic input of the spectrometers.

The laser atomic-emission multichannel spectrometer developed here is in active use in scientific research, as well as for teaching at the laser physics and spectroscopy department of the physics faculty of the Belarusian State University. The technical characteristics of the developed spectrometer match those of modern worldwide equipment for laser atomicemission spectroscopy with two-pulse excitation. The technical implementation and associated program have not only enabled scientific studies with single- and double-pulse laser ablation and supported the development of a method for quantitative and layer-by-layer microanalysis of a wide class of objects, but also enabled the use of a laser atomic-emission multichannel spectrometer for teaching.

\section{REFERENCES}

1. E. Grifoni, S. Legnaioli, M. Lezzerini, G. Lorenzetti, S. Pagnotta, and V. Palleschi, J. Spectrosc., 849310(1-5) (2014). 
2. W. Bauer, G. P. Perram, and T. Haugan, J. Appl. Phys., 123, 1-11 (2018).

3. M. Corsi, G. Cristoforetti, M. Hildalgo, D. Iriatre, S. Legnaioli, V. Palleschi, A. Salvetti, and E. Tognoni, Appl. Spectrosc., 57, No. 6, 715-721 (2003).

4. V. S. Burakov, A. F. Bokhonov, M. I. Nedel'ko, and N. V. Tarasenko, Kvant. Élektron., 33, No. 12, 1065-1071 (2003).

5. L. Radziemski and D. Cremers, Spectrochim. Acta: At. Spectr., 87, 3-10 (2013).

6. E. S. Voropay, K. F. Ermalitskaia, and F. A. Ermalitskii, J. Appl. Spectrosc., 86, No. 2, 294-299 (2019).

7. L. Joliveta, M. Leprince, S. Moncayo, L. Sorbier, C.-P. Lienemann, and V. Motto-Ros, Spectrochim. Acta: At. Spectr., 151, 41-53 (2019).

8. Kh. Bazzal, A. R. Fadaiyan, and A. P. Zazhogin, Zh. Bel. Gos. Univ. Fizika, No. 1, pp. 34-42 (2017).

9. M. P. Patapovich, Zh. I. Buloichik, I. D. Pashkovskaya, N. I. Nechipurenko, and A. P. Zazhogin, Zh. Bel. Gos. Univ. Fizika, No. 2, pp. 27-33 (2017).

10. Zh. I. Buloichik, A. P. Zazhogin, N. I. Nechipurenko, M. P. Patapovich, and I. D. Pashkovskaya, Zh. Bel. Gos. Univ. Fizika, No. 1, pp. 9-17 (2018).

11. K. F. Ermalitskaia, Y. S. Voropay, and A. P. Zazhogin, J. Appl. Spectrosc., 77, No. 2, 153-159 (2010) .

12. E. S. Voropai, I. M. Gulis, K. F. Ermalitskaya, F. W. Ermalitskii, K. N. Kaplevskii, A. E. Rad'ko, and K. A. Shevchenko, Pribory Tekhn. Éksperim., 4, 154-155 (2016). 